\title{
Wireless Charging Technology Based on Photovoltaic PowerGeneration and Its Application
}

\author{
Maoyan Fan ${ }^{1}$, Lifang Zhang ${ }^{2, a}$ \\ ${ }^{1}$ School of Physicsand Electronic Engineering, Yuxi Normal University, Yuxi 653100, Yunan Province, China \\ ${ }^{2}$ Office of Academic Affairs, Yuxi Normal University, Yuxi 653100, Yunan Province, China
}

\begin{abstract}
A new technology of wireless charging based on the photovoltaic power generation micro-grid is designed with the combination of photovoltaic array and battery to ensure the reliability of power supply. This research aims at the transmission principle of the series-parallel (SP) wireless charging technology via coupled magnetic resonances and the relationships of power, frequency, distance and efficiency. The charging strategy integrating maximum power point tracking with four-stage charging is achieved. The Boost circuit with $\mathrm{XC164CM}$ single chip microcontroller as the core is designed and fabricated for the charging experiments. The results show that this method can charge quickly, avoid overcharge and realize optimum system running, thus having broad application prospects and high promotion value.
\end{abstract}

\section{Introduction}

The wireless charging technology is a new type of charging method that achieves the energy transmission through electromagnetic effect and realizes no electrical connection between the charger and the load device. Compared with the conventional charging method, it is free from the constraint of cable and thus flexible and convenient. The magnetically coupled resonant wireless power transmission system has the advantages of high transmission power and long transmission distance ${ }^{[1-2]}$. The "Mas Three Laws" can be applied with the integration of the charging current and the maximum power point tracking of the photovoltaic array. The combined power supply of the photovoltaic cells and the battery can maintain the efficiency and stability of the charging of the system. The application of micro-grid and wireless charging technology to the electric vehicle charging system can effectively reduce the impact of electric vehicle charging on the power grid and improve the economic and environmental benefits of the photovoltaic power generation system ${ }^{[3]}$.

There are three types of wireless power transmission methods: electromagnetic induction, magnetic resonance coupling and microwave radiation. In 2007, the Massachusetts Institute of Technology "lighted up" a $60 \mathrm{~W}$ light bulb from a distance more than $2 \mathrm{~m}$ away; the University of Auckland in New Zealand studied the online wireless power transmission technology based on Double-D as transmitter coil and developed the world's first wireless charging bus by cooperation with the Conductix-Wampfler from Germany; the Oak Ridge National Laboratory of the United States conducted research on coupling mechanism, transmission characteristics and dielectric loss of wireless charging. In
China, Chongqing University, Southeast University and Harbin Institute of Technology researched magnetically coupled resonant wireless power transfer ${ }^{[4-5]}$. Research work mainly focuses on the study of circuit topologies and coils to improve the transmission power and efficiency.

\section{The Principle of Wireless Charging}

The wireless charging system realizes the energy transfer from the transmitter coil to the receiver coil according to the principle of electromagnetic induction, including power supply, high-frequency inverter, energy transmitter coil, energy receiver coil, rectifier and load. The impedance of the energy receiver coil is equivalent to the energy transmitter coil to obtain a simplified circuit model. The transmitter and receiver coils are wound with Litz wires and contain multiple thin copper wires to effectively reduce the resistance caused by the skin effect of the current, thereby reducing system losses. Reducing the loss resistance value on the two coils can improve the transmission efficiency of the wireless charging system. The loss resistance values on the two coils include resistance losses of the coils, eddy current loss induced by the alternating magnetic field and hysteresis loss in ferromagnetic materials. The key technologies of the wireless charging are described as follows. (1) The magnetic shielding design of the coupling mechanism reduces the influence of the wireless charging coupling mechanism on the electromagnetic environment in the non-working area and improves the coupling efficiency. (2) To solve the problems of close proximity and weak offset of the inductive coupling structure and susceptibility of the resonant coupling structure to interference, we combine the coupling coefficient with

${ }^{\mathrm{a} C o r r e s p o n d i n g ~ a u t h o r: ~ f f @ y x n u . n e t ~}$ 
the quality factor in the design to improve the load quality factor of the receiver coil and enhance the offset compatibility.

The principle of magnetic resonance coupling-based wireless charging is that the circuits at two sides have the same resonant frequency. When the circuits resonate, the transmitter and receiver coils can achieve the wireless power transmission by resonance coupling of the spatial magnetic field. At present, the methods for analyzing magnetic resonance coupling-based wireless charging are roughly divided into two types: coupled-mode theory and equivalent circuit theory. The coupled-mode theory analyzes from the perspective of system energy, skips complex physical models and constructs an accurate, simple and intuitive analysis architecture. The equivalent circuit theory builds a circuit model between the transmitter coil and the receiver coil, finds out the equivalent relationship according to the mutual inductance theory and Kirchhoff's laws and solves the system. It is the most widely applied methods ${ }^{[6]}$. The equivalent circuit theory is used to analyze the wireless charging based on the magnetic coupling resonance SP. As shown in Fig. 1, $U_{S}$ is the high-frequency power supply, $R_{S}$ represents the internal resistance of the power supply, $L_{1}$ and $L_{2}$ are the inductances of the transmitter coil and the receiver coil, respectively, $M$ denotes the mutual inductance of the two coils, $C_{1}$ and $C_{2}$ are the resonant capacitances at the transmitter end and the receiver end, $R_{1}$ and $R_{2}$ are the resistances at the transmitter end and the receiver end, and $R_{L}$ is the equivalent load. For the convenience of calculation, it is designed as $L_{1}=L_{2}=L \quad ; \quad C_{1}=C_{2}=C \quad$; $R_{1}=R_{2}=R$. When the power frequency supplied by the power supply to the transmitter coil is the resonant frequency, the transmitter coil continuously transmits energy to the receiver coil and finally to the load. At the resonant frequency, the equation $K V L$ of the model is:

$$
\left\{\begin{array}{l}
\left(R+R_{S}\right) I_{1}-j \omega M I_{2}=U_{S} \\
-j \omega M I_{1}+R I_{2}-I_{3} / j \omega C=0 \\
\left(1 / j \omega C+R_{L}\right) I_{3}-I_{2} / j \omega C=0
\end{array}\right.
$$

Here: $\omega$ - the natural angular frequency of the system. $I_{1}$ and $I_{3}$ are obtained from Eq.(1). When they are substituted into the output power $P_{L}=I_{3}^{2} R_{L}$, the input power $P_{i n}$, the output power $P_{\text {out }}$ and the working efficiency $\eta$ of the wireless transmission system can be calculated. If the wireless charging system is operating in the resonance state, that is, when $\omega=1 / \sqrt{L_{1} C_{1}}=1 / \sqrt{L_{2} C_{2}}$, the transmission efficiency of the system reaches the highest, then:
$P_{L}=\frac{\omega^{4} C^{2} M^{2} U_{S}^{2} R_{L}}{\left[R+R_{S}-j \omega^{3} C M^{2} B-j \omega C R\left(R+R_{S}\right) B\right]^{2}}$

Transmission efficiency:

$\eta=\frac{\omega^{4} C^{2} M^{2} R_{L}}{R+R_{S}-j \omega^{3} C M^{2} B-j \omega C R\left(R+R_{S}\right) B}$

According to Eqs.(2) and (3), the output power and the transmission efficiency are multivariable functions of natural angular frequency $\omega$, mutual inductance $M$, capacitance $C$, load $R_{L}$, loss resistance $R$ and internal resistance $R_{S}$ of the power supply. When the circuit parameters are determined, the output power and the transmission efficiency are only related to the natural angular frequency $\omega$ and the mutual inductance $M, \omega$ is related to the frequency and $M$ is related to the transmission distance $D$, and therefore the relationship between output power, transmission efficiency, frequency and transmission distance can be obtained.

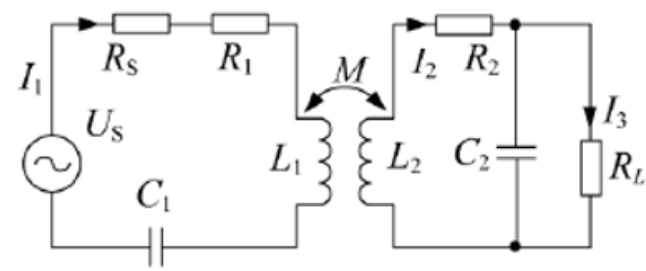

Fig.1 magnetic resonance coupling

The equivalent circuit of the wireless power transmission system is shown in Fig.2. It has the characteristics that the resonant frequency is not affected by the coupling coefficient of the primary side and the secondary side and it is easy to realize the high-power transmission. In the figure, $L_{1}$ and $R_{1}$ are the inductance and resistance of the primary side, $C_{r 1}$ is the resonant capacitance which resonates with $L_{1}$ and the frequency value is equal to the inverter switching frequency. Therefore, the resulting impedance of $L_{1}$ and $C_{r 1}$ is zero.

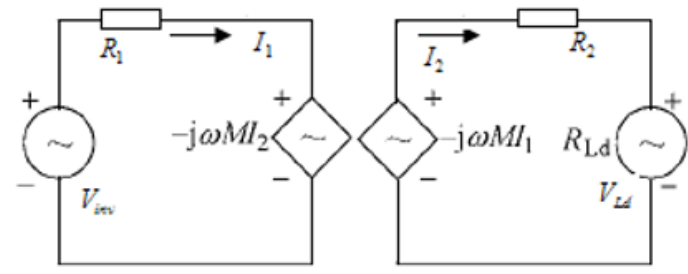

Fig.2 Equivalent circuit

According to the Kirchhoff's Voltage Law ( $K V L$ ) and the Ohm's Law, the following equation can be obtained:

$$
\begin{gathered}
V_{i n v}=\frac{1}{j \omega C_{r 1}} I_{1}+j \omega L_{1} I_{1}+R_{1} I_{1}-j \omega M I_{2} \\
V_{L d}=j \omega M I_{1}-j \omega L_{2} I_{2}-\frac{1}{j \omega C_{r 2}} I_{2}-R_{2} I_{2}
\end{gathered}
$$

Here, $M$ is the mutual inductance between the primary side and the secondary side, which is small because there is a large gap. The equivalent circuit of the wireless power transmission system is in a resonant state. 
The inductance and the capacitance offset with each other. There is only resistance in the equivalent circuit. The circuit equation is as follows:

$V_{\text {inv }}=R_{1} I_{1}-j \omega M I_{2} ; V_{L d}=j \omega M I_{1}-R_{2} I_{2}$

Therefore, the application of resonant capacitance can eliminate the inductances $L_{1}$ and $L_{2}$, and the overall impedance of the system is also significantly reduced. In the design of the wireless power transmission system, the output frequency of the high-frequency inverter and the natural resonant frequency of the coils are consistent, thereby achieving the efficient power transmission. The optimum design of transmitter and receiver coils is at the core of the design of the wireless power transmission system. They need to maintain the same natural resonant frequency and have a high $Q$ value. The system structure design involves the mutual influence of multiple factors such as coil diameter, wire diameter, material and perimeter, so the parameter optimization is critical.

\section{Design of Charge Controller System}

In the independent photovoltaic (PV) power generation system, it is difficult for a single charging control method to satisfy the four-stage charge. After the integrated application of the advantages of constant current charge and constant voltage charge in the independent PV power generation system, it has the following functions: (1) the maximum power output of the system is achieved by the maximum power point tracking; (2) when the input power of the photovoltaic array is insufficient, the battery discharges to supply power to the wireless charging load battery; when the photovoltaic output power is sufficient, priority is given to the wireless charging load battery; (3) the battery protection function can protect the battery from overcharge and over discharge and prolong its service life; (4) the display, alarm, and communication functions enable the working condition parameter communication with the main controller of the photovoltaic power generation system. The DC power output from photovoltaic cells is stored in the energy storage device through the control circuit. The energy storage device can store the photoelectrically converted electrical energy, provide electrical energy for the wireless charging circuit transformation and realize the wireless power transmission.

\subsection{System Hardware Design}

The charging control system consists of Buck circuit, PWM drive circuit, data acquisition circuit, and maximum power point tracking control circuit. The specific procedure is detailed as follows: the Buck converter achieves the charging conversion from the photovoltaic array to the battery pack; the data acquisition circuit collects the current and voltage signals of the photovoltaic array and those of the battery pack during charging and discharging. The collected signals are transmitted directly to the main controller for A/D conversion. In the main controller, the duty cycle of the
PWM signals is controlled according to the maximum power point tracking (MPPT) algorithm. Then signals are transmitted to the drive circuit to control the state of the power switch and enable the reasonable charging control of the system.

The coils at primary sides and secondary sides of the transmitter module and the receiver module for electromagnetic energy conversion are wound by Litz wires of the same type ${ }^{[7]}$. In the experiment, the distance of the two coils is fixed to improve the system stability. Through the adjustment of the transmitter circuit parameters, the power amplification effect is maximized and the voltage peak at the signal output end is increased. Since the inductance value of the transmitter coil has been determined due to the influence of the coil material and the winding method, the series compensation capacitor of the transmitter circuit can be adjusted only on condition that other circuit parameters remain unchanged, so as to achieve the maximum output voltage and ensure that the output signal waveform isn't distorted. The energy transmitter and receiver circuit is shown in Fig.3.

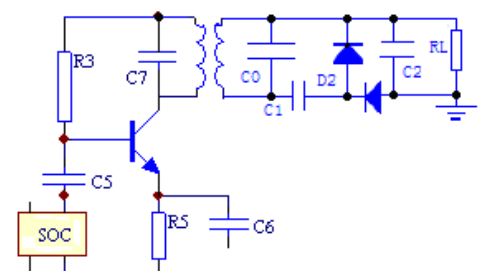

Fig.3 Energy transmitter and receiver circuit

When the system transmission efficiency is tested, the main testing system efficiency varies with the load change and the change of distance between transmitter coil and receiver coil. For the parallel resonant circuit, the wire diameter of the air-core coupling coil is $0.5 \mathrm{~mm}$, the diameter is $10 \mathrm{~cm}$, the inductance is $47 \mu \mathrm{H}$ and the carrier frequency is $8 \mathrm{MHz}$. The input voltage at the transmitter end and the effective value of current are measured in the experiment, and the input power of the system is calculated. For testing the receiver power, $R_{L}$ is the adjustable load resistance. By testing the voltage attached to both ends, the output power of the system is calculated and finally the system output efficiency may be calculated ${ }^{[8]}$.

\subsection{System Software Design}

The software system includes charging control program and data acquisition program. The charging control program is composed of intelligent charging program and photovoltaic MPPT control program. The data acquisition program comprises current sampling program, voltage sampling program and direct memory access (DMA) data processing program. It has the following functions. (1) The intelligent charging program detects the battery state and determines the working condition of the photovoltaic charging controller. It is divided into three sub-programs: maximum power charge, over-charge and float charge, to ensure high-efficiency operation of the system. (2) The PWM program is a driving pulse signal that generates 
two complementary dead time controls. According to the charging state of the system, on-time of the Buck circuit is controlled by changing the duty cycle of the PWM to realize the photovoltaic charging control. (3) The data acquisition program samples the input voltage and current of PV arrays and those of battery packs through the ADC port, and stores them by DMA mode to achieve the high-speed and accurate sampling and provide data guarantee for the control program.

In the photovoltaic power generation system, the battery charge and discharge control technology can directly influence the system performance. The charging process of the charger is divided into four stages: shorttime trickle charge, constant current charge, constant voltage equalization charge and float charge.

(1) Short-time trickle charge. Charging starts with the stable small current to help activate reactive materials in the battery and avoid damage to the battery caused by the high-current charge. This stage is controlled by the single-chip microcontroller to start the timing circuit.

(2) Constant current charge. According to the Mas Curve, the acceptable current is relatively large at the initial stage of charging and the battery is charged at the maximum power at this stage to ensure the system efficiency. The battery has relatively large constant current for charging. If the photovoltaic power generation is insufficient, the maximum charging current is smaller than $I_{b s}$. In order to improve the charging efficiency, a reasonable solution is switching to the constant current charge by the battery power supply. The key technique is the judgment criteria for switching between the PI constant current charging algorithm and the MPPT charging algorithm.

(3) Constant voltage charge. After the maximum power charge stage, the battery capacity can reach about $80 \%$. In order to further make the battery reach the full capacity, we must charge the battery at a constant voltage. Similar to the constant current charge, the given voltage may not be maintained when the sunlight is insufficient and it is also necessary to switch from the PI constant voltage charging algorithm to the MPPT charging algorithm. The switching criteria are similar to those of constant current charge, but the control object is switched to the charging voltage.

(4) Float charge. In the float charge stage, the battery capacity is a dynamic balancing process. The float charge is used to supplement energy consumed by the battery self-discharge, which can effectively prevent the battery capacity loss due to undercharge and prolong the service life of the battery.

The intelligent charging strategy can be flexibly utilized and the battery conditions can be analyzed according to the terminal voltage of the connected battery. The system determines which charging state it proceeds to for realizing the intelligent charging strategy. The MPPT algorithm is added to the charging control to ensure the efficiency of the photovoltaic system utilizing solar energy resources, so that the charging becomes reasonable and fast and the life of the battery is prolonged.

\section{Application of Charging System}

The photovoltaic micro-grid wireless charging system consists of three parts: photovoltaic power generation system, wireless power transmission system and electric vehicle charging system. The experimental system structure is shown in Fig.4.

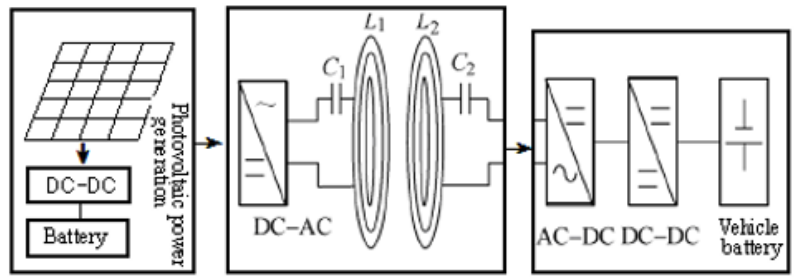

Fig.4 Overall structure of charging of electric vehicles

The photovoltaic micro-grid power generation system can effectively solve the problem that the photovoltaic power generation is influenced by sunlight and temperature change. The photovoltaic system is directly connected to the DC bus. The battery is connected to the DC bus via the bidirectional DC-DC. The maximum power point tracking of the $\mathrm{PV}$ and the charging and discharging of the battery are conducted by the DC-DC on the battery side. When the electric vehicle is charged in the daytime, the photovoltaic maximum power point tracking voltage $V_{M P P}$ is used as the reference voltage for the DC-DC voltage boost, and the photovoltaic power and the battery synergize to achieve the maximum photovoltaic power output.

In the photovoltaic system, the battery is frequently charged and discharged and shows some new features. (1) Due to the randomness, finiteness and intermittent nature of photovoltaic power generation and the randomness of load demand, it is difficult for photovoltaic power to charge according to specific charging laws. (2) The continuous rainy days are likely to cause deep discharge of the battery. Based on the stage charging strategy of battery, combined with the characteristics of the photovoltaic system, together with the maximum power point tracking control of the photovoltaic array, the charging method integrating the maximum power charge and the stage charge is adopted, so that the charging and discharging process of the battery is applicable to the independent photovoltaic system ${ }^{[9]}$.The specific charging control procedure is shown in Fig. 5.

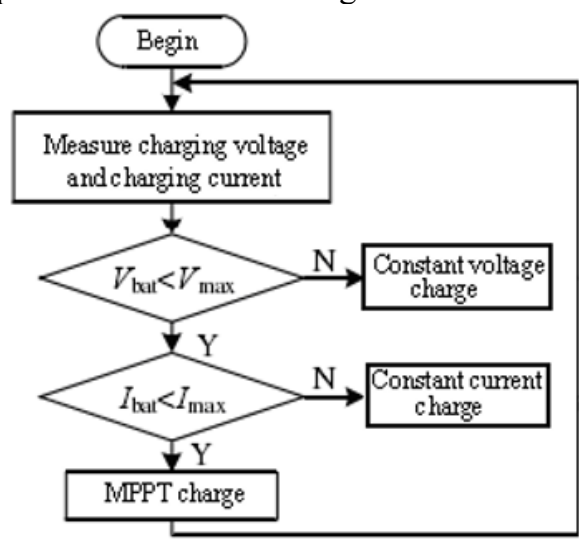

Figure 5 Photovoltaic power generation charging strategy flow chart 
The experimental results show that the power transmission efficiency increases first and then decreases with the increase of the distance, and the transmission efficiency is the highest at the distance of $30 \mathrm{~cm}$, reaching $80 \%$. The power transmission efficiency of the experimental system is not always the maximum at the resonant frequency, but at the critical coupling. In addition, the distance between transmitter coil and receiver coil is changed from far to near and the output frequency of the signal generator is adjusted so that the system transmission efficiency is maximized at each distance and the efficiency-distance curve of the frequency tracking shown in Fig. 6 is obtained. It can be known from the tracking curve that when the distance is smaller than the distance to the critical coupling point, even if the coupling factor becomes smaller as the distance increases, the transmission efficiency does not change much; when the distance is larger than the distance to the critical coupling point, even if the frequency tracking is performed, the transmission efficiency will drop rapidly, so the maximum distance of the wireless power transmission is the distance to the critical coupling point ${ }^{[10]}$.

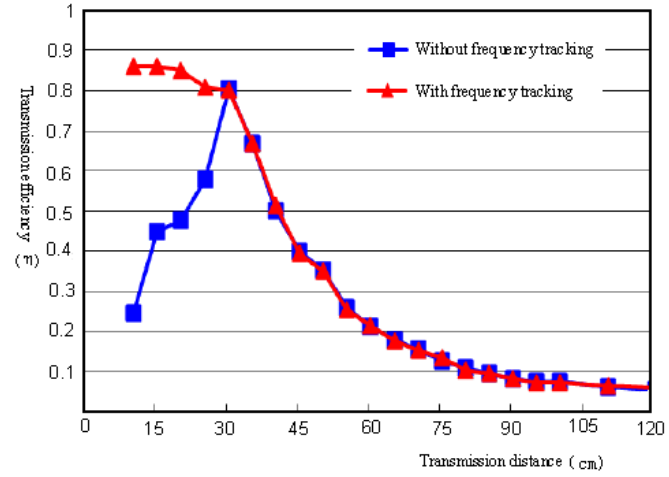

Fig.6 Efficiency-distance characteristic curve with/without frequency tracking

As revealed by the experiment, the four-stage charge based on the discharge pulse integrates the MPPT algorithm to coordinate the charging control method of photovoltaic cell modules and lead-acid battery, which can improve the working efficiency of the system and enable the absorption of more electric energy by the leadacid battery and the efficient utilization of photovoltaic cell modules and lead-acid battery.

\section{Conclusions}

A new green environmentally friendly charge system is designed by integrating the photovoltaic power generation with the wireless charging technology. The MPPT algorithm is applied to coordinate the relationship between the load battery and the photovoltaic module, improve efficiency, meet actual needs of some special applications and expand the application field of wireless charging technology ${ }^{[11]}$. The wireless power transmission technology via coupled magnetic resonances can achieve the charging strategy combining MPPT and four-stage charge and the judgment of battery conditions by setting the reference voltage in stages. The experiment demonstrates the feasibility of the MPPT wireless charging in the independent solar photovoltaic conversion system, which can improve the wireless charging efficiency of the photovoltaic power generation.

\section{Acknowledgments:}

We are grateful for the support of the scientific research fund of Yunnan Provincial Department of education (Project number: 2015Y412)!

We thank the support from Undergraduate Innovation and Entrepreneurship Training Program of Yunnan Province (Project number: 2016A03)!

\section{References}

1. Liao Chenglin,Li Junfeng,Wang Lifang,et al.MidRange Wireless Charging System for Electric Vehicle. Transactions of China Electrotechnical Society,2013, 28(S2): 81-85.

2. Bosshaxd R. and J W Kolar. Multi-Objective Optimization of $50 \mathrm{~kW} / 85 \mathrm{kHz}$ IPT System for Public Transport[J].IEEE Journal of Emerging and Selected Topics in Power Electronics,2016, 4(4SI):1370-1382.

3. Ibrahim M,et al.Inductive Charger for Electric Vehicle:Advanced Modeling and Interoperability Analysis [J].IEEE Transactions on Power Electronics, 2016,31(12): 8096-8114.

4. Guo Chun. Research and Design of Magnetically Coupled Resonant Wireless Charging System[D]. North University of China, 2017.

5. Ding Weidong. Research on High Power Wireless Power Transfer via Magnetic Coupled Resonant [D]. University of Electronic Science and Technology of China, 2015.

6. Zhang Xian,Yang Qingxin,Chen Haiyan et al. Modeling and Design and Experimental Verification of Contactless Power Transmission Systems via Electromagnetic Resonant Coupling[J].Proceedings of the CSEE, 2012,32 ( 21):153-158.

7. Huang Hui,Huang Xueliang,Tan Lin-lin,et al. Research on transmitter and receiver of wireless power transmission based on magnetic resonance coupling [J].Advanced Technology of Electrical Engineering and Energy. 2011, (01): 32-35.

8. Liu Zhizhen,Zeng Hao,Chen Hongxing,Hei Tong, Zhou Bo. Design and optimization of magnetic core structures for EV wireless charging systems [J]. Electric Machines and Control, 2018,22(1):8-15.

9. De Brito M A G,L, Sampaio L P, et al. Evaluation of the Main MPPT Techniques for Photovoltaic Applications[J].Industrial Electronics IEEE Transactions on, 2013, 60(3):1156-1167.

10. Li Yang. Method and Experimental Study for High Power Wireless Power Transfer via Coupled Magnetic Resonances [D]. Hebei University of Technology, 2012. 
11. Miller J M,Jones $\mathrm{P}$ T,Li J M,et al. ORNL

Experience and Challenges Facing Dynamic Wireless Power Charging of EV's[J]. IEEE Circuits\&Systems Magazine, 2015,15(2):40-53. 